\title{
Evidence for Sequential Decision Making in the Medicinal Leech
}

\author{
Teresa Esch, ${ }^{1}$ Karen A. Mesce, ${ }^{2}$ and William B. Kristan ${ }^{1}$ \\ 1Section of Neurobiology, Division of Biological Science, University of California, San Diego, La Jolla, California 92093, \\ and ${ }^{2}$ Departments of Entomology and Neuroscience, University of Minnesota, St. Paul, Minnesota 55108
}

\begin{abstract}
Decision making can be a complex task involving a sequence of subdecisions. For example, we decide to pursue a goal (e.g., get something to eat), then decide how to accomplish that goal (e.g., go to a restaurant), and then make a sequence of more specific plans (e.g., which restaurant to go to, how to get there, what to order, etc.). In characterizing the effects of stimulating individual brain neurons in the isolated nervous system of the leech Hirudo medicinalis, we have found evidence that leeches also make decisions sequentially. In this study, we describe a pair of interneurons that elicited locomotory motor programs, either swimming or crawling, in isolated nerve cords. In semiintact animals, stimulating the same neurons also produced
\end{abstract}

either swimming or crawling, and which behavior was produced could be controlled experimentally by manipulating the depth of saline around the intact part of the leech. These same neurons were excited and fired strongly when swimming or crawling occurred spontaneously or in response to mechanosensory stimulation. We conclude that these brain interneurons help to decide on locomotion (i.e., they are "locomotory command-like neurons") and that the ultimate behavior is determined downstream, in a part of the decision-making hierarchy that monitors stimuli related to the depth of fluid surrounding the leech.

Key words: choice behavior; leeches; neural circuits; motor patterns; multifunctional neurons; locomotion
Animals continually assess their surroundings and make behavioral adjustments to act appropriately under current conditions. An animal selects a goal, on the basis of internal drives and external conditions, that helps it to survive (Tinbergen, 1951). Sometimes animals must choose between two conflicting goals: a hungry animal might abandon eating to avoid being captured by a predator. Different tasks can be used to achieve a selected goal, and each task can take different forms (Stein et al., 1986). For example, to escape predation an animal could locomote through water or over land. Locomotion over land could take several forms, such as crawling or running. An animal must use sensory information to inform its decisions about which form of which task to use if it is to achieve its current goal. How these decisions are made should be reflected in the neural architecture. At one extreme, decisions about goal, task, and form may be made independently, at separate neural locations. At the other extreme, sensory information could trigger one form of a task directly, with no overt choice about goal or task ever being made.

Because invertebrate nervous systems are more accessible experimentally and have fewer neurons than vertebrate brains, they can yield insight into the mechanisms of behavioral choice that are difficult to study in higher vertebrates (Glimcher, 2001; Schall, 2001). Although invertebrates surely do not engage in the cognitive processes that human introspection considers "decision making," they do exhibit the behavioral manifestation of choice: they predictably do one thing or another, in one way or another, in response to complex stimuli. Therefore, even simple organisms engage in a process akin to decision making. Understanding the

Received Aug. 20, 2002; revised Oct. 4, 2002; accepted Oct. 9, 2002.

This work was supported by National Institutes of Health (NIH) Grants MH43396 and NS35336 (W.B.K.), National Research Service Award MH12029 (T.E.), NIH Training Grant NS07220 (T.E.), and a University of Minnesota Career Development Grant (K.A.M.).

Correspondence should be addressed to William B. Kristan, University of California San Diego, Neurobiology Section, Division of Biological Science 0357, 9500 Gilman Drive, La Jolla, CA 92093-0357. E-mail: wkristan@ucsd.edu.

Copyright (C) 2002 Society for Neuroscience 0270-6474/02/2211045-10\$15.00/0 neuronal events underlying simple behavioral choices can help us understand decision making in higher animals.

The leech is an excellent model system for studying behavioral choice. Leeches exhibit simple reflex behaviors, including local bending (Lockery and Kristan, 1990) and whole-body shortening (Shaw and Kristan, 1995), as well as complex, modifiable behaviors such as swimming (Kristan et al., 1974) and crawling (Eisenhart et al., 2000). These behaviors can be elicited, and their circuit-level interactions can be studied in both isolated nerve cords and semi-intact preparations. Such studies have indicated that each of several leech neurons is active in multiple behaviors. The mechanisms that effect switching between behaviors have begun to be described (Shaw and Kristan, 1997).

Several neurons capable of initiating behavior have somata located in the leech's subesophageal ganglion and possess axons that project the entire length of the nerve cord (Brodfuehrer and Friesen, 1986; Brodfuehrer and Burns, 1995; Brodfuehrer et al., 1995b). To date, all identified command-like neurons in the subesophageal ganglion either initiate or terminate swimming. Studies of shortening, however, have suggested that commandlike neurons for this behavior also are located in the subesophageal ganglion (Shaw and Kristan, 1999). These observations suggest that the subesophageal ganglion may contain neurons capable of initiating several behaviors.

In this study, we located candidate decision-related neurons in the subesophageal ganglion of the leech by retrograde labeling of long-distance projection neurons. In a preliminary report (Esch and Kristan, 2002), we described one of these neurons, which initiates both swimming and crawling motor patterns in isolated nerve cords. We now present more extensive data, using semiintact preparations, that confirm the previous data and demonstrate that which behavior the neuron elicits can be controlled experimentally by changing the saline level in the recording chamber. These data support a serial model of behavioral choice in which the decision to locomote (the task) is made before the decision about what form of locomotion to produce. 


\section{MATERIALS AND METHODS}

\section{Animals}

Adult Hirudo medicinalis weighing 2-5 gm were obtained from Leeches USA (Westbury, NY) or Zaug (Biebertal, Germany) and were maintained in artificial pond water at $15^{\circ} \mathrm{C}$. The general experimental methods were as described previously (Kristan et al., 1974).

\section{Preparations}

Isolated nerve cord preparation. Leeches were anesthetized in chilled leech saline (Nicholls and Purves, 1970). The entire nerve cord, including the supra-esophageal and subesophageal ganglia, the 21 midbody ganglia, the tail brain, and the interganglionic connectives, were dissected from the leech. Dorsal posterior (DP) nerves were dissected away from the body tissue and left attached to the ganglia in one to two segments, usually between ganglia 7 and 16 . The ventral blood sinus was dissected away from the subesophageal ganglion, one to two midbody ganglia, and one to two sections of connective. The remainder of the sinus was left in place. The nerve cord was pinned in a Sylgard-coated $60 \mathrm{~mm}$ tissue culture dish and bathed in leech saline. The sheath covering the subesophageal ganglion was removed, as was the sheath covering the segmental ganglia when segmental interneurons were recorded. In some cases, the leech nerve cord was transected posterior to ganglion 17 or 18 , with no apparent effect on the results.

Semi-intact preparation. Leeches were anesthetized in chilled leech saline. A small incision was made near the posterior sucker, and nerves innervating the sucker were severed to prevent its attachment to the substrate. A second incision was made in the second annulus of segment 4. The incision was extended anteriorly to expose the nerve cord, which was dissected from the surrounding tissue, including the ventral sinus, anterior to ganglion 4 . The DP nerves of segment 3 were dissected from the body wall and left attached to the ganglion, and the nerves of ganglion 4 were severed. Much of the connective and muscle tissue within segment 3 was then removed, and the entire body wall anterior to segment 3 was removed. This resulted in a mostly intact preparation with the subesophageal ganglion and first three ganglia exposed, and a flap of denervated skin attached to the anterior end. The flap of skin was used to pin the preparation in a wax-filled, rectangular recording chamber. The chamber was filled with leech saline to various levels as follows. In "low" saline most of the body of the leech was exposed to air, but the nerve cord was submerged. In "high" saline levels, the entire leech was submerged. Generally, in "intermediate" levels the leech was submerged when fully elongated, but the leech would emerge partially from the saline when it was swimming or when it was fully shortened. Because the size of leeches varied, the absolute levels of saline in each category also varied.

Denervated leeches. For some behavior experiments, nerves to the head brain were severed. The leeches were anesthetized with saline containing $8 \%$ ethanol. The skin dorsal to the head brain was opened, and nerves were severed. Care was taken to minimize damage to surrounding tissue. The skin was then sutured closed with surgical threads, and leeches were allowed to recover for at least $24 \mathrm{hr}$ in artificial pond water.

\section{Behavior of intact and denervated leeches.}

To study the effects of water level on the form of locomotion produced by intact and denervated leeches, leeches were monitored in a Plexiglas behavior arena measuring $49 \times 4.5 \times 7 \mathrm{~cm}$ (length $\times$ width $\times$ height) and containing different amounts of water $(0-1000 \mathrm{ml})$. Six leeches were tested on 3-4 d over a 2 week period, both before and after denervation (described above). Before placing a leech in the arena, we manually agitated the leech to promote activity. At each water level tested, we attempted to induce the leech to locomote across the length of the arena at least four times. If a leech did not locomote, it was prodded with a wooden dowel. Occasionally a leech would not locomote at all and would be removed from the chamber. Leeches were removed from the arena before the water level was changed.

The different forms of locomotory behaviors were quantified as a proportion of the total number of locomotory episodes. A locomotory episode was defined as a locomotory movement beginning with front sucker detachment and ending with rear sucker placement (Cacciatore et al., 2000). Therefore, each step of crawling was considered a locomotory episode, whereas searching movements were excluded. A swim movement was considered a swim/crawl step if it resulted in locomotion less than half the length of the arena, with subsequent sucker attachment and body shortening; otherwise, it was classified as "swim." The proportion of behaviors in each category for each water level was calculated indepen- dently for each leech for each day of testing. If the leech failed to locomote during the session, zero was entered for all three categories.

\section{Electrophysiology and behavior}

Connective and DP nerve recordings were made with glass-tipped suction electrodes. Connectives were recorded en passant between two ganglia, generally between four and five in isolated cords and between two and three for semi-intact preparations. Intracellular recordings were made with sharp microelectrodes filled with $3 \mathrm{~mm}$ potassium acetate, having resistances of $40-50 \mathrm{M} \Omega$. In most experiments, $\sim 2.5 \%$ Neurobiotin (Vector Laboratories, Burlingame, CA) was dissolved in the solution in the recording electrode to aid in subsequent morphological identification of the impaled cell (see below).

Cell selection. The ventrolateral circular excitor (CV) was identified by its position (Stuart, 1970) and firing pattern (Eisenhart et al., 2000). The paired neuron R3b1 was identified by the following characteristics. (1) The cell body was located in the R3b packet, just medial and slightly anterior to a consistently located prominent cell of unknown function. (2) Intracellular spikes were matched one-to-one with spikes in the connective. (3) Depolarizing the neuron with 2-4 nA of current elicited the crawling motor pattern. (4) The neuron, when filled with an intracellular dye, had a distinctive morphology. Four morphologically distinct neurons were routinely recorded, and each was uniquely correlated with a distinct behavior; however, whenever a neuron in this region elicited crawling, it had a morphology similar to that shown in Figure $1 b$. In $\sim 75 \%$ of the experiments, R3b1 was correctly selected by its position alone, as confirmed subsequently by the physiological and morphological criteria.

Monitoring of behaviors. In isolated nerve cords, fictive behaviors were monitored by examining the firing patterns of the dorsal excitor motor neuron cell 3 in the DP nerve(s) and of CV neurons. The motor patterns for different behaviors are distinctive, as described previously (Kristan et al., 1974; Shaw and Kristan, 1995; Eisenhart et al., 2000). In semi-intact preparations, direct observation of the intact portion of the leech was used in addition to a DP recording to monitor behaviors. When swimming occurred, bursts of cell 3 firing indicated the dorsal contraction phase of each cycle. When crawling occurred, however, cell 3 bursts in the anterior segments were not sufficient to indicate the contraction phase: we found that cell 3 sometimes fired during elongation in these segments, presumably to assist in lifting the head. Because the cell 3 firing pattern was insufficient to indicate the phase of crawling, a foot pedal was used to mark the times at which elongation and contraction waves were observed to begin.

Behaviors were elicited in the semi-intact preparation by using thin wooden applicator sticks to stroke or prod the anterior or posterior end of the leech. We found that these probes, of all the stimulation techniques tried, produced the smallest artifact. Timing of the stimulation was marked with a foot pedal. In isolated cord preparations, electrical stimulation was applied to the DP nerves via a Grass stimulator (10 msec pulses delivered at $10 \mathrm{~Hz}$ for $500 \mathrm{msec}$ ).

Data acquisition. Physiological data were digitized with a MacADIOS A-D board and displayed and analyzed with Superscope II (GW Instruments, Somerville, MA). To compute the spike-triggered average, Superscope data were imported into Matlab (Mathworks, Natick, MA).

Spike analysis. Because the spike-initiating zones of most projection neurons in the leech are located far from the soma, the spikes measured in the soma are small $(<5 \mathrm{mV})$ and can be difficult to distinguish from the noise. Therefore, to measure the spike frequency, spikes were counted by manually comparing the intracellular recording with the connective recording. When R3b1 was firing at low frequency in an inactive leech, spikes in the connective were easily identifiable by their latency from intracellular spikes (see Fig. 1c). When more activity was present, spikes could be identified by size, shape, and latency. The time of each spike was recorded and used to make raster plots or histograms.

\section{Neuronal labeling}

Retrograde labeling. The head brain and anterior three to four ganglia were dissected from the leech and placed in leech saline. A small well, made from a round section of a plastic pipette tip and Vaseline, was built around the cut end of the connective. In some cases, the well surrounded only one lateral connective, so that ipsilaterally and contralaterally projecting neurons could be distinguished. The well was filled with distilled water, and crystals of rhodamine dextran (molecular weight, 3000; Molecular Probes, Eugene, OR) or Neurobiotin were added to a final concentration of $>5 \%$. Dye was allowed to diffuse for $2-3 \mathrm{~d}$ at $4^{\circ} \mathrm{C}$, after 

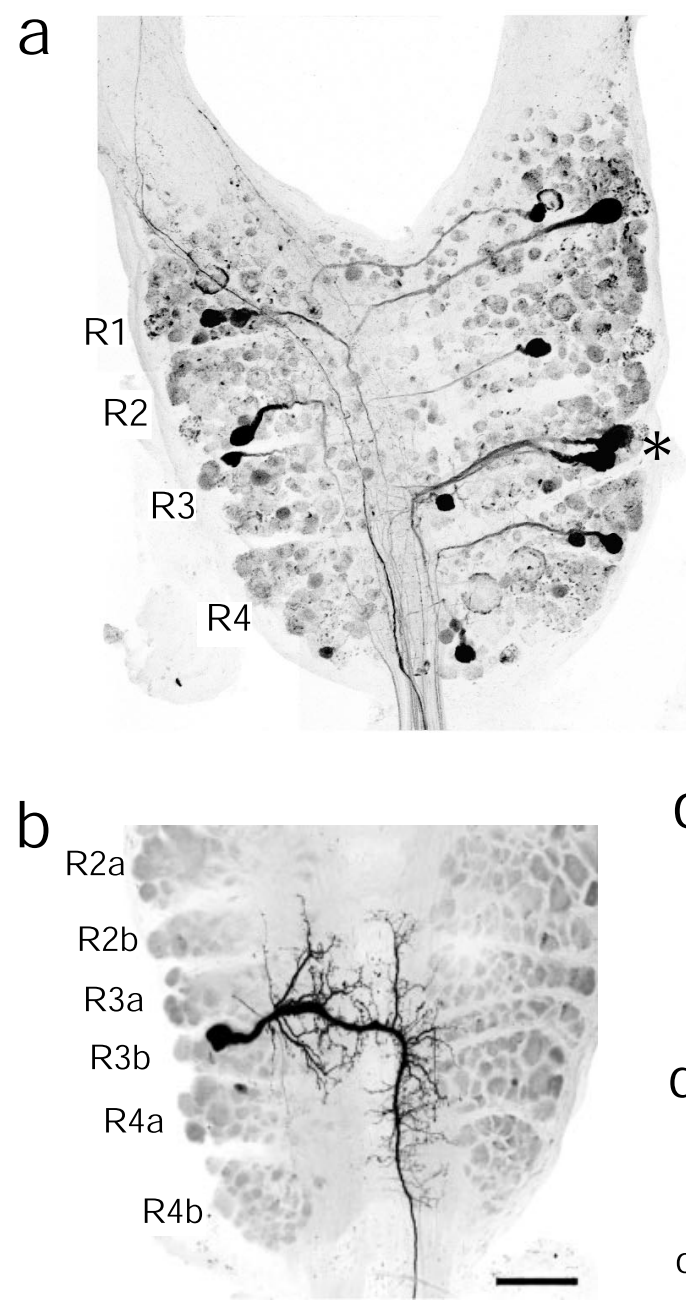

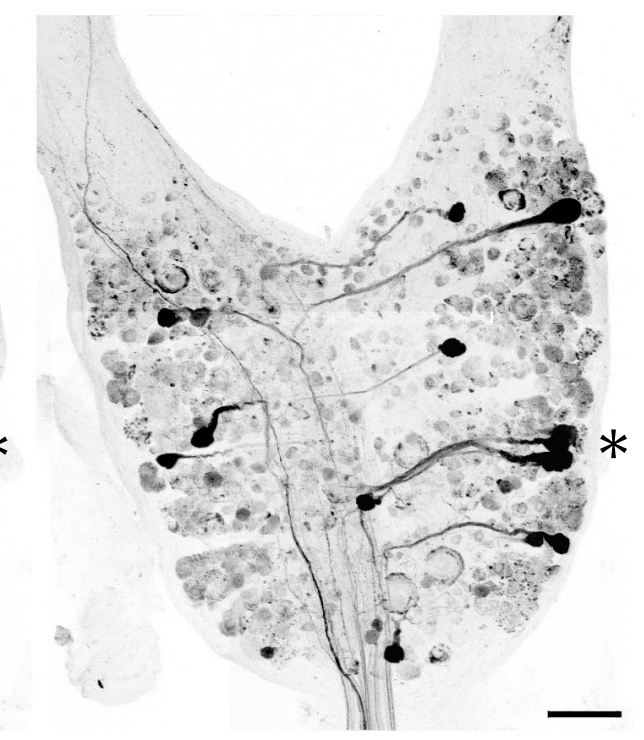

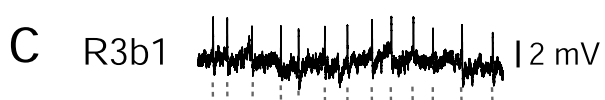

conn $4 / 5$

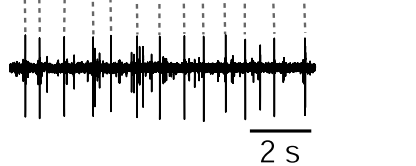

d

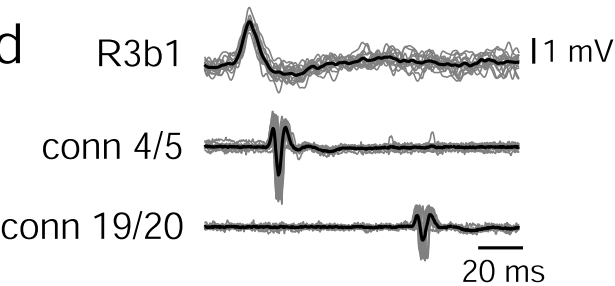

Figure 1. Localization and morphology of cell R3b1. $a$, Stereomicrographs of projection neurons in the subesophageal ganglion. The left lateral connective was placed in a well of concentrated rhodamine dextran, and dye diffused retrogradely to the head brain. The rostral neuromeres (i.e., packets homologous to four segmental ganglia) $R 1-R 4$ are labeled. The asterisk marks a cell cluster in $\mathrm{R} 3 . b$, The soma of $\mathrm{R} 3 \mathrm{~b} 1$ was filled with Neurobiotin during electrophysiological recording and then labeled with $\mathrm{Cy} 3$ streptavidin. Subpackets of the subesophageal neuromeres are labeled. Scale bar: $a, b, 100 \mu \mathrm{m}$. $c$, Illustration of spike matching in intracellular (top) and connective (bottom) recordings. Dashed lines connect intracellular spikes to the corresponding spikes in the connective, measured between ganglia 4 and 5 . These traces were used to make the spike-triggered average in $d$. $d$, Electrical recordings showing superimposed spikes in R3b1 recorded intracellularly (top trace) and extracellularly (en passant) in the connective between ganglia 4 and 5 (middle trace) and between ganglia 19 and 20 (bottom trace). The intracellular signal was filtered at $300 \mathrm{~Hz}$. The preparation was bathed in $20 \mathrm{~mm}$ $\mathrm{Mg}^{2+} / 0 \mathrm{mM} \mathrm{Ca}^{2+}$ saline to eliminate synaptic activity. A spike-triggered average (black trace) was calculated from 17 individual traces (gray traces). which the preparations were fixed with $2 \%$ paraformaldehyde in PBS. If Neurobiotin was used for retrograde labeling, tissue was permeabilized with $0.3 \%$ Triton X-100 and incubated with Cy3-conjugated streptavidin (Jackson ImmunoResearch, West Grove, PA). After rinsing, and regardless of the dye used, tissue was dehydrated through a series of ethanol dilutions and cleared with methyl salicylate. Tissue was mounted in Gurr DePeX mounting medium and imaged with a Zeiss laser scanning confocal microscope using Bio-Rad software (Hercules, CA).

Intracellular fills. Cells were iontophoretically injected with either Neurobiotin or rhodamine dextran. In most cases, the dye was included in the recording electrode, but sometimes the cell was reimpaled with a dye-filled electrode after electrophysiological recordings. Dye was passed into the cell with alternating pulses of positive and negative current (0.5-1.5 nA). Dye was allowed to diffuse in the cell for 30-90 min, after which the tissue was processed and imaged as described above.

\section{RESULTS}

To locate the somata of projection neurons that might govern behavioral selection, we labeled subesophageal ganglion neurons of the leech by retrograde transport of rhodamine dextran through the ventral nerve cord. This method indicated the presence of a cluster of cells in the third packet on the dorsal surface of the rostral brain (Fig. 1a, R3). Therefore, we focused on this region in subsequent experiments. In this paper, we describe a bilateral pair of neurons in the posterior subpacket of R3 (R3b), and we refer to each neuron of the pair as R3b1.

R3b1 has a distinctive morphology that can be used to identify the cell in different preparations (Fig. 1b). Its soma lies centrally in the R3b packet and has short anterior-projecting neurites both ipsilateral and contralateral to the cell body. Its axon projects in the lateral connective on the contralateral side. By matching intracellular action potentials to spikes in the connective recordings, we found that the axon projects the entire length of the nerve cord (Fig. $1 c, d$ ). In 10 preparations in which nearby neurons were recorded and filled along with R3b1, each cell could be distinguished easily by its morphological features. Furthermore, as will be discussed below, this neuron could be uniquely identified by its ability to elicit crawling behavior. Every time current injection into a soma in the R3b packet was able to elicit crawling, that neuron had the morphology of R3b1 (34 preparations). In contrast, no neuron that had a different morphology was able to elicit crawling (34 cells, exhibiting three different morphologies). Therefore, R3b1 is morphologically and functionally distinguishable from other neurons in the R3b packet.

In 22 of 24 isolated nerve cord preparations (Fig. $2 a$ ), passing positive current into an R3b1 neuron elicited a crawling-like motor pattern (Fig. $2 b$ ), which is consistent with our preliminary report (Esch and Kristan, 2002). The alternating bursts that occur in the dorsal longitudinal motor neuron cell 3 and in the circular motor neuron CV (Fig. 2b) would result in contraction and elongation, respectively, in an intact leech (Eisenhart et al., 2000). The bursts of action potentials in cell 3 produced after current injection into cell R3b1 progressed along the length of the leech, anterior to posterior, as they do in crawling (Esch and Kristan, 2002). Fictive crawling elicited by electrical stimulation of R3b1 

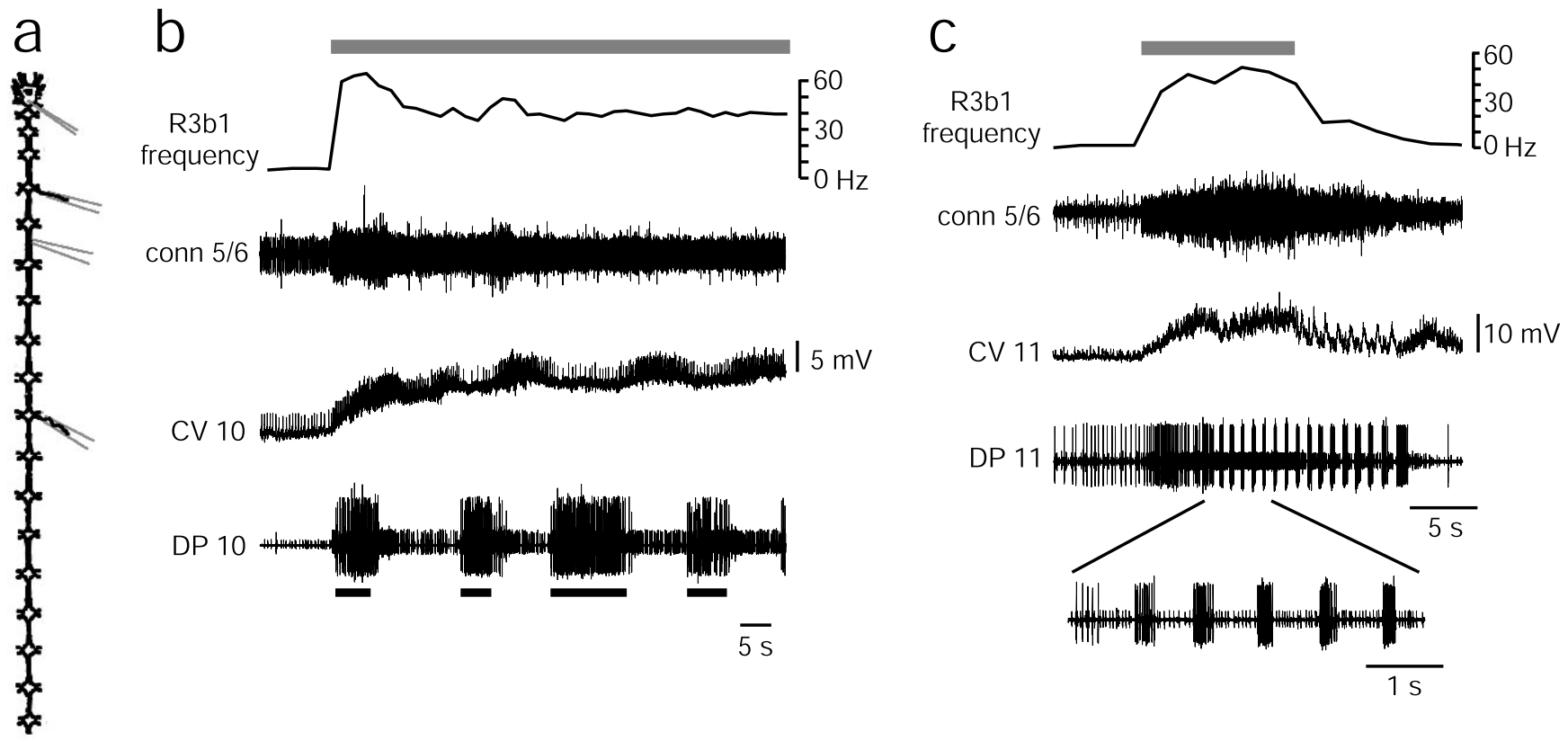

Figure 2. Current injection into R3b1 elicits either the crawling or the swimming motor pattern. In isolated nerve cords, 3-4 nA of current was injected into R3b1 during the times indicated by gray bars at the top of each set of traces. Current injection caused increased spiking of R3b1, as monitored in the extracellular connective (conn 5/6) recording in each trace. Spikes were counted manually, and the spike frequency (measured in 2 sec bins) is indicated in each set of traces. $a$, Schematic drawing of the isolated nerve cord preparation. $b$, Intracellular recording of a CV neuron and extracellular recording of a DP nerve exhibit the crawling motor pattern. Black bars below DP trace indicate time of fictive contraction, based on peak firing of cell 3. $c$, Stimulation of R3b1 in a different preparation produced approximately the same firing frequency in R3b1 but elicited the swim motor pattern. CV and cell 3 spikes (in the DP recording) increase in frequency and then occur as bursts as swimming begins. In this case, swimming continued for many cycles after current injection ceased. Note that the period of swimming is much shorter than that of crawling. A portion of the DP trace has been expanded to show details of the burst pattern in cell 3.

normally continued throughout the period of stimulation, and sometimes it persisted for two or more cycles after termination of the stimulus. We conclude, therefore, that activation of R3b1 can elicit the crawling motor pattern.

In some preparations, depolarizing current injected into R3b1 elicited the swimming motor pattern rather than the crawling pattern (Fig. 2c), confirming preliminary results (Esch and Kristan, 2002). Swimming often lasted only as long as current was injected, as happened with crawling, but it was not uncommon for the swimming motor pattern to outlast electrical stimulation by many swim cycles. Usually, when the swimming motor pattern was elicited, the crawling motor pattern was elicited by previous or subsequent stimulation of the same cell. In 24 preparations, a neuron identified morphologically as R3b1 was stimulated; in 11 of these, R3b1 elicited swimming in some trials and crawling in others. There was only one preparation in which R3b1 stimulation elicited swimming but not crawling, whereas in another 11 preparations only crawling was produced. (In the remaining preparation, a cell with the morphology of R3b1 did not elicit a recognizable motor pattern.) Therefore, R3b1 activation reliably elicits two different locomotory motor patterns: swimming and crawling.

To gain a clearer understanding of what factors determine which behavior is elicited by activating R3b1, we used a semiintact preparation (Fig. 3a). Because segments 5-20 were intact, we could determine the behavioral response to electrical stimulation of R3b1 simply by observing the animal. In addition, with the semi-intact preparation we could assess the effects of tactile sensory stimulation on R3b1. These experiments suggested that sensory feedback is important for determining whether stimulation of R3b1 elicits swimming or crawling. In low saline levels, current injected into R3b1 elicited the crawling motor pattern
(Fig. $3 b)$ ( $n=5$ leeches). When the saline level was raised, similar stimulation of the same R3b1 elicited swimming (Fig. 3c). Therefore, R3b1 appears to be a state-dependent locomotory neuron; which locomotory behavior it elicits depends on sensory information about the fluid level.

Although electrical stimulation of R3b1 elicited apparently normal swimming and crawling in high and low saline levels, respectively, in intermediate saline levels it elicited a hybrid behavior (Fig. 3d). The leech elongated and contracted with a rhythm typical of a normal crawl, but rather than elongating steadily as in normal crawling, the leech produced swimming undulations while elongating. This hybrid behavior also was produced by freely moving, fully intact leeches in intermediate water levels (see below). Nonetheless, the behavior was more consistent and occurred over a broader range of fluid levels in the semiintact preparation when the front of the leech was pinned to the substrate (see Materials and Methods). The swim/crawl hybrid was the most common form of locomotory behavior observed in the semi-intact preparation, probably because of the depth of the chamber and the fact that the brain was dissected away from the surrounding tissue (see below).

Direct observation of the semi-intact leech during electrical stimulation of $\mathrm{R} 3 \mathrm{~b} 1$ revealed that, regardless of which behavior was elicited, the initial motor response was elongation. This can be seen in Figure 3, $b$ and $d$, in which elongation always precedes contraction, as well as in Figure 2, $b$ and $c$, in which the circular motor neuron $\mathrm{CV}$ is activated immediately after R3b1 stimulation. Elongation was followed by contraction when either crawling or the swim/crawl hybrid behavior was elicited, but contraction never occurred first, even if the leech was relatively elongated at the time of stimulation. Elongation also occurred before the onset 


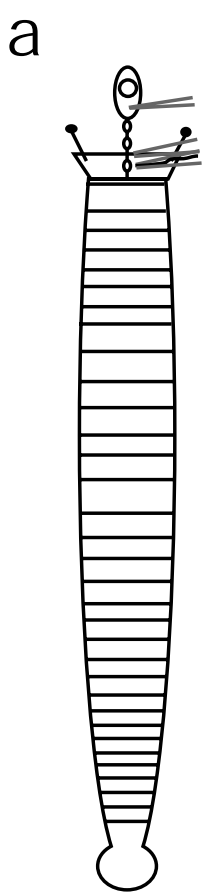

b
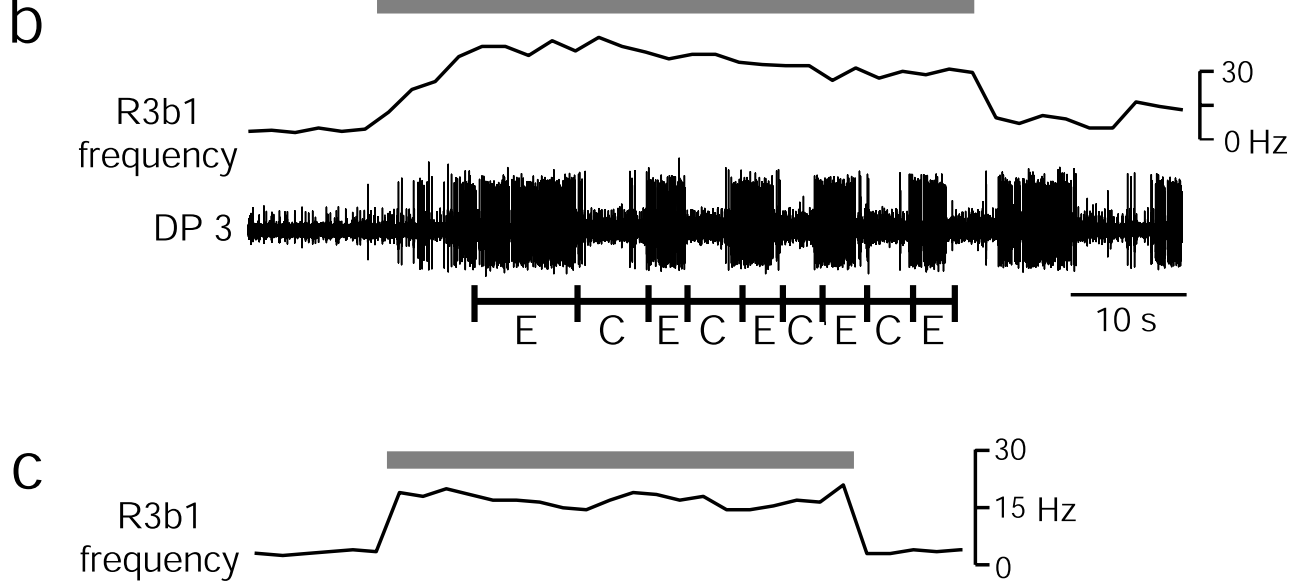

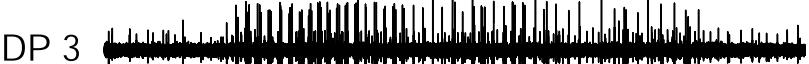
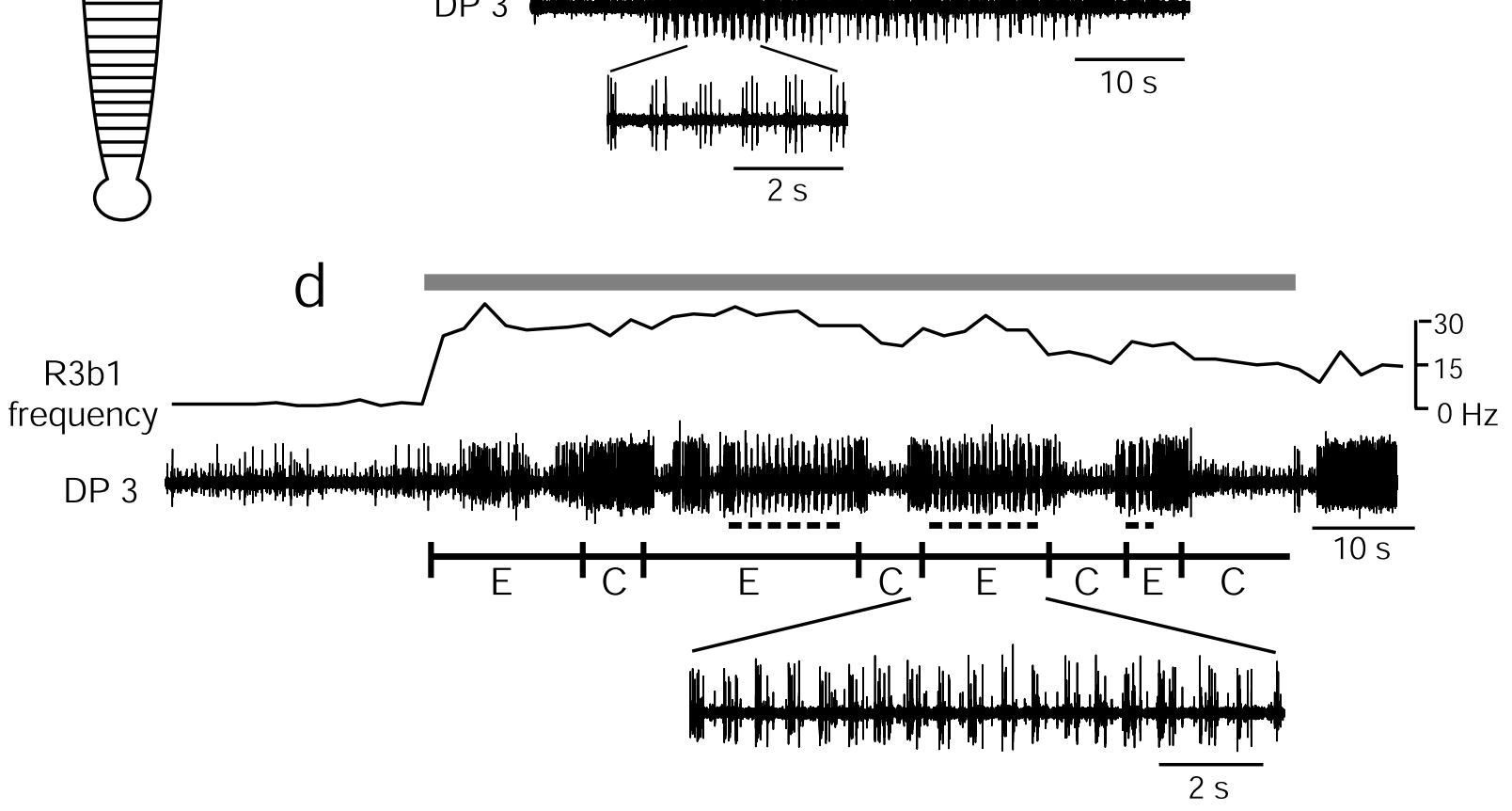

Figure 3. Effect of electrical stimulation of R3b1 in semi-intact preparation depends on the saline level in the chamber. Current (3-4 nA) was injected into R3b1 for the times indicated by gray bars at the top of each set of traces in $b-d$. The spike frequency of R3b1 was determined by counting spikes in a connective recording and grouping them into $2 \mathrm{sec}$ bins. Behaviors were observed directly and also recorded in the activity of the DP nerve in segment 3. The beginning of elongation $(E)$ and contraction $(C)$ were marked with a foot pedal and are indicated below the traces. $a$, Schematic drawing of semi-intact preparation. $b$, In low saline levels, current injection elicited crawling. In this example, cell 3 bursts occur in DP 3 during elongation, probably to assist in raising the head. $c$, After the saline level was increased, electrical stimulation of R3b1 elicited swimming in the same preparation. $d$, In intermediate saline levels, stimulation of R3b1 elicited a hybrid behavior in which the leech swam (dots below DP trace) during elongation. In $c$ and $d$, a portion of the trace has been expanded to show details of the swim bursts.

of dorsal-ventral undulation when swimming or the hybrid behavior was elicited by R3b1 stimulation. Furthermore, as illustrated in Figure $2 c, \mathrm{CV}$ fired at a high rate throughout the electrical stimulation of R3b1, which implies that elongation actively occurred even after swimming had begun. Finally, weak electrical stimulation of R3b1 ( $\sim \mathrm{nA})$ often elicited elongation without producing a full swim or crawl motor pattern (data not shown). Therefore, it appears that the initial role of R3b1 in behaviors is to produce elongation.

The form of locomotion produced by intact leeches was affected by water levels similarly to what we observed in semi-intact leeches (Fig. 4a). When the behavioral arena was a moistened Plexiglas sheet, leeches always crawled. When water was present in the arena, leeches always began a locomotory episode by elongating. Even in the shallowest water tested $(3 \mathrm{~mm})$, leeches would sometimes make swimming movements after elongating. Leeches were unable to produce swimming movements without both emerging from the water and striking the floor of the arena in water depths of $<10 \mathrm{~mm}$, and in these shallower water depths initial swimming movements resulted in a swim/crawl hybrid. In water $10 \mathrm{~mm}$ deep, leeches could swim only by turning on their sides; when they did not do this, swim movements still resulted in the hybrid behavior. (Note that the depth of the recording chamber used for semi-intact preparations was $10 \mathrm{~mm}$, and therefore our finding that semi-intact leeches often produced the hybrid behavior is consistent with our observations using intact leeches.) In water levels deeper than $10 \mathrm{~mm}$, whenever swimming undulations were produced they resulted in full swims that usually 
Figure 4. Choice of locomotory behavior depends on water level and is altered by severing head brain nerves. The proportion of all locomotory behaviors belonging to each category ( $\mathrm{crawl}$, swim/crawl, or swim) is plotted as a function of water level. $a$, Intact leeches $(n=6$ leeches; each tested three times). $b$, The same leeches as in $a$, after all nerves to the head brain had been severed ( $n=3$ leeches; each tested three times). Error bars are SEM.
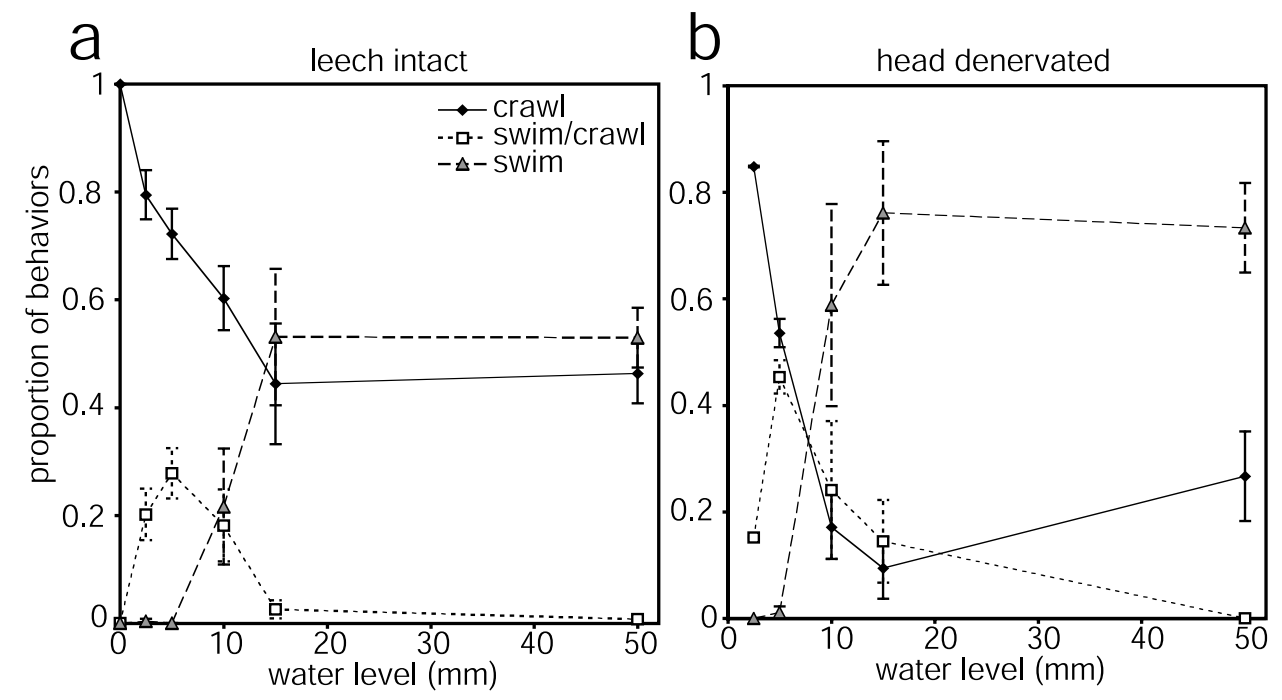

continued until the leech reached the far end of the arena. The hybrid behavior was never produced in these deeper water levels, which is consistent with previous findings that once a swim is initiated it will usually continue until the leech contacts an obstacle (Kristan et al., 1974). Still, even in the deepest water levels, $\sim 50 \%$ of locomotory events were crawl steps (Fig. $4 a$ ), indicating that water depth is only one factor determining whether leeches swim or crawl.

The decision to swim or crawl is influenced by information carried by head brain nerves. After recording six intact leeches as described above, we exposed the head brain and cut zero to two of the most anterior nerves in three leeches and all seven head brain nerves in the remaining three leeches. The skin was sutured, and the leeches were allowed to rest for $2 \mathrm{~d}$ before being retested in the arena. Leeches in which zero to two nerves had been cut behaved similarly to intact leeches (data not shown). In contrast, leeches in which all head brain nerves were cut were much more likely to attempt swimming movements than intact leeches (Fig. $4 b$ ). In water depths of $\geq 6 \mathrm{~mm}$, the proportion of behaviors that included swimming movements was $\sim 50 \%$ greater in denervated leeches than in intact leeches (compare Fig. 4, a and $b$ ). Denervated leeches often would raise their heads $1-2 \mathrm{~cm}$ out of the water while attempting to swim, something rarely seen in intact leeches. These data, along with the increased occurrence of the swim/crawl hybrid in semi-intact leeches described above, suggest that some of the information determining the type of locomotion produced in different water levels is carried by head brain nerves.

In addition to activating both swimming and crawling when artificially stimulated with current injection, R3b1 became depolarized when either of these behaviors was elicited by mechanical stimulation of the intact portion of the leech in semi-intact preparations. The ongoing activity pattern of R3b1 differed during the two behaviors, however (Fig. 5a,b). During crawling, the membrane potential of R3b1 oscillated $5-10 \mathrm{mV}$ in phase with each crawl step (Fig. 5a): R3b1 depolarized and increased spiking during the onset of elongation, the membrane potential returned to baseline as contraction began, and another increase in spiking of R3b1 heralded the onset of the next elongation wave at the front of the leech. This oscillatory pattern was also observed in R3b1 after electrical stimulation was terminated, in those cases in which crawling continued after the stimulation (data not shown).
Oscillations and spiking of R3b1 were also observed during crawling motor patterns produced in isolated nerve cords (data not shown). In contrast to this oscillating membrane potential during crawling, the membrane potential of R3b1 remained depolarized 5-10 $\mathrm{mV}$ above the resting membrane potential throughout a swimming episode, returning to baseline only when swimming stopped (Fig. $5 b$ ). In $\sim 50 \%$ (12 of 26 ) of swimming bouts, a small oscillation at the swim frequency was seen on top of the tonic depolarization level. This oscillation was present in both isolated and semi-intact preparations and was not consistently present or absent in a single preparation. During the swim/crawl hybrid, the activity of R3b1 was oscillatory, similar to that in crawling, with occasional oscillations in phase with swimming around the elongation depolarization (data not shown). Therefore, R3b1 is phasically active during crawling and mostly tonically active during swimming, and in both cases R3b1 fires most when the leech is elongated.

Natural sensory stimuli that evoked swimming or crawling, such as nudging or lifting the posterior end of the leech, led to depolarization and spiking in R3b1. Stimuli that were too weak to evoke a locomotory response produced a small, short-lived depolarization of R3b1 (Fig. $5 c$ ) ( $n=4$ of 6 stimulations), whereas stimuli sufficient to initiate locomotion produced a larger and longer lasting depolarization of R3b1 (Fig. 5a,b) ( $n=12$ of 15 stimulations in eight leeches). Other sensory stimuli that evoked swimming or crawling, such as changing the saline level or shining a light on the animal, also produced a depolarization of R3b1 (data not shown). Furthermore, depolarization of R3b1 accompanied spontaneous swimming and crawling episodes (Fig. 5d). These data strongly suggest that R3b1 contributes to the initiation of swimming and crawling in response to natural stimuli.

The different activity patterns recorded in R3b1 during crawling versus swimming do not appear to be the sole determinant of which behavior is produced. If this were the case, then steady current injection into the cell should always elicit swimming. As described above, this is clearly not the case: crawling often was produced during sustained electrical stimulation of R3b1 (Figs. $2 b, 3 a)$.

The firing frequency of R3b1 does not appear to influence the decision to swim or to crawl. Swimming and crawling were both elicited over a broad range of R3b1 firing frequencies. When current injection into R3b1 elicited crawling, the mean firing rate 

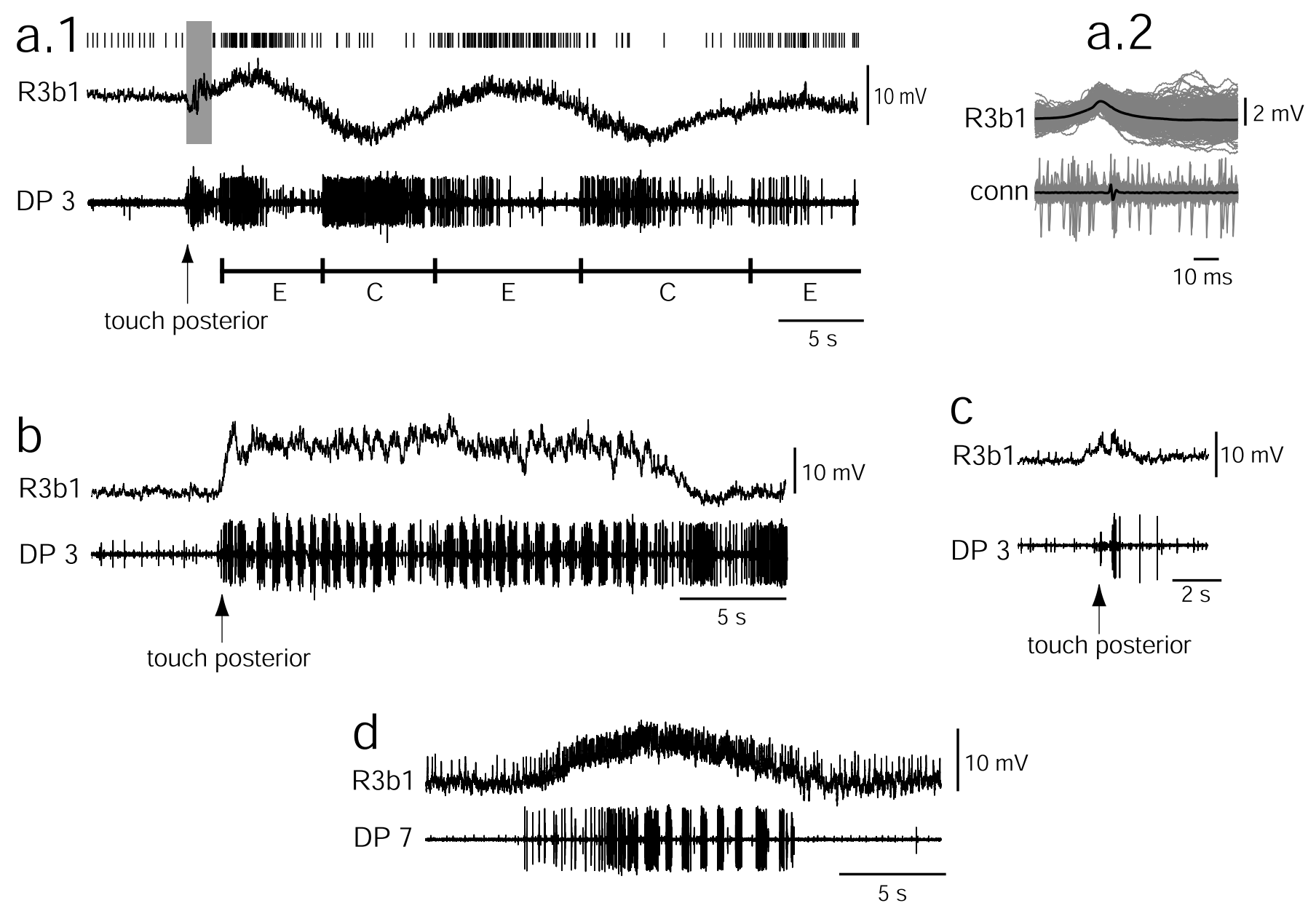

Figure 5. R3b1 is active during naturally elicited crawling and swimming. In $a-c$, the posterior end of the leech was briefly prodded (arrows) with a wooden applicator stick to elicit behaviors. a.1, Touch-elicited crawling. R3b1 was activated and spikes occurred (raster above traces) while the leech elongated. $E$ and $C$ (below traces) indicate the onset of elongation and contraction, as determined by direct observation. Stimulus-associated noise in the intracellular recording precluded accurate spike counting during the time indicated by the gray box. a.2, Average (black) of traces (gray) centered on spikes marked in $a .1$, showing that intracellular spikes (top) align with spikes in the connective (bottom). To simplify the figure, the connective recording is not shown in $a .1 . b$, Swimming was elicited. In this preparation, an oscillation at the swim frequency occurred about the depolarized level. $c$, A weak stimulation of the posterior leech elicited a small depolarization of R3b1, but no overt behavior. $d$, R3b1 became depolarized and spiked at an increased frequency throughout a spontaneous swim bout.

of R3b1 was $37 \pm 2 \mathrm{~Hz}$, and when swimming was elicited the mean firing rate of R3b1 was not significantly different $(39 \pm 2$ $\mathrm{Hz}$ ). Furthermore, depolarization of R3b1 during an ongoing locomotory behavior never resulted in a switch from swim to crawl or vice versa. Instead, depolarizing current injection during a swim episode increased the intensity of swimming, whereas depolarization during crawling increased the rate of crawling steps (data not shown). These data suggest that factors other than the activity pattern and firing frequency of R3b1 must be involved in the decision to swim or to crawl.

In contrast to sensory stimuli that evoke locomotion, stimuli that halt locomotion or evoke an incompatible behavior hyperpolarize R3b1. In an inactive, semi-intact leech, touching segment 5 elicited a whole-body shortening response, accompanied by a sharp hyperpolarization of R3b1 (Fig. $6 a)(n=14$ of 18 stimulations in eight leeches). The same stimulus applied to a swimming leech stopped the swimming, evoked shortening, and brought the membrane potential of R3b1 back to baseline (Fig. $6 b)$. The activity of R3b1 in response to this mechanical stimulation depended on the behavior produced, however. For example, after a second, similar stimulus in Figure $6 a$, the leech elongated after an initial shortening response. In this case, R3b1 became depolarized and began spiking after the initial hyperpolarization. Therefore, the activity of R3b1 is correlated more strongly with the behavior produced than with the stimulus given.

\section{DISCUSSION}

In this study, we describe a paired neuron, R3b1, that elicits both crawling and swimming in the medicinal leech. Which behavior $\mathrm{R} 3 \mathrm{~b} 1$ activity produces is influenced by sensory input and can be manipulated in the semi-intact preparation by changing fluid levels in the recording chamber. Although there are other leech neurons that participate in multiple behaviors (e.g., 204, Tr1, and SE1) (Brodf uehrer and Friesen, 1986; Kristan et al., 1988; Brodfuehrer et al., 1995a; Shaw and Kristan, 1997), this is the first example of a neuron in the leech that clearly activates two different behaviors in a context-dependent manner.

On the basis of our results, we propose a working hypothesis for the circuit underlying the choice to swim or crawl in the leech (Fig. 7). Stimulation of pressure-sensitive mechanoreceptors ( $\mathrm{P}$ cells) in the leech's posterior activates R3b1 neurons (Fig. 5). $\mathrm{R} 3 \mathrm{~b} 1$ neurons, in turn, activate a network (E), including the 

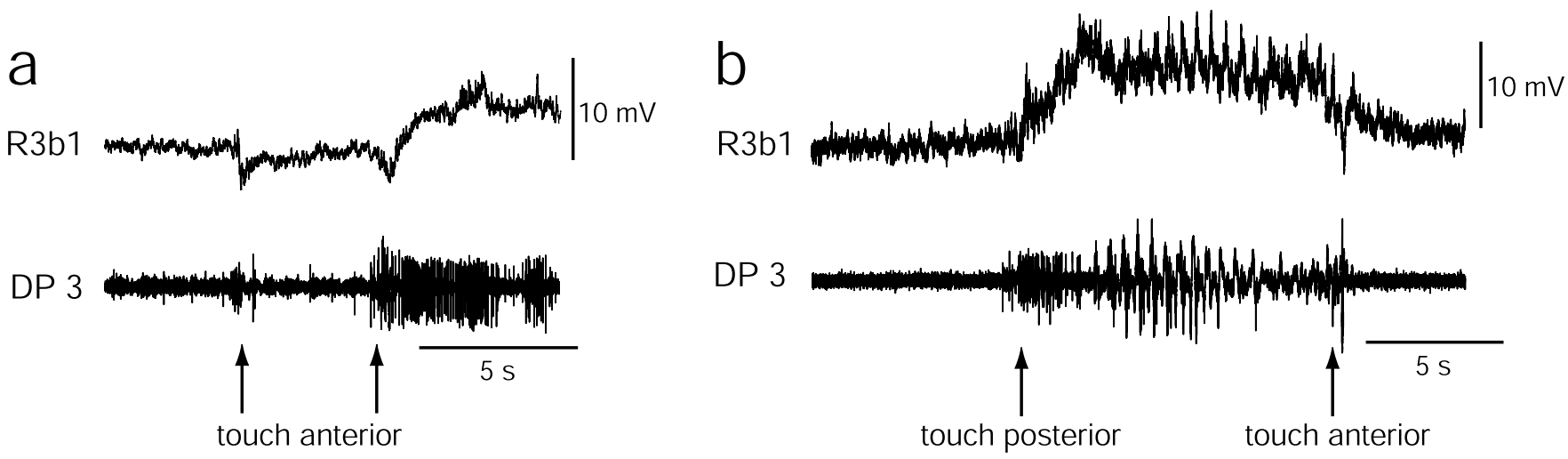

Figure 6. R3b1 is inhibited by stimuli that cause shortening. $a$, In an inactive leech, prodding the anterior portion of the leech near segment 5 (arrows) elicited shortening. After a second, similar stimulus, the leech elongated. $b$, Touching the posterior of the leech ( first arrow) elicited swimming. A subsequent touch to the anterior of the leech (second arrow) stopped swimming and elicited shortening.

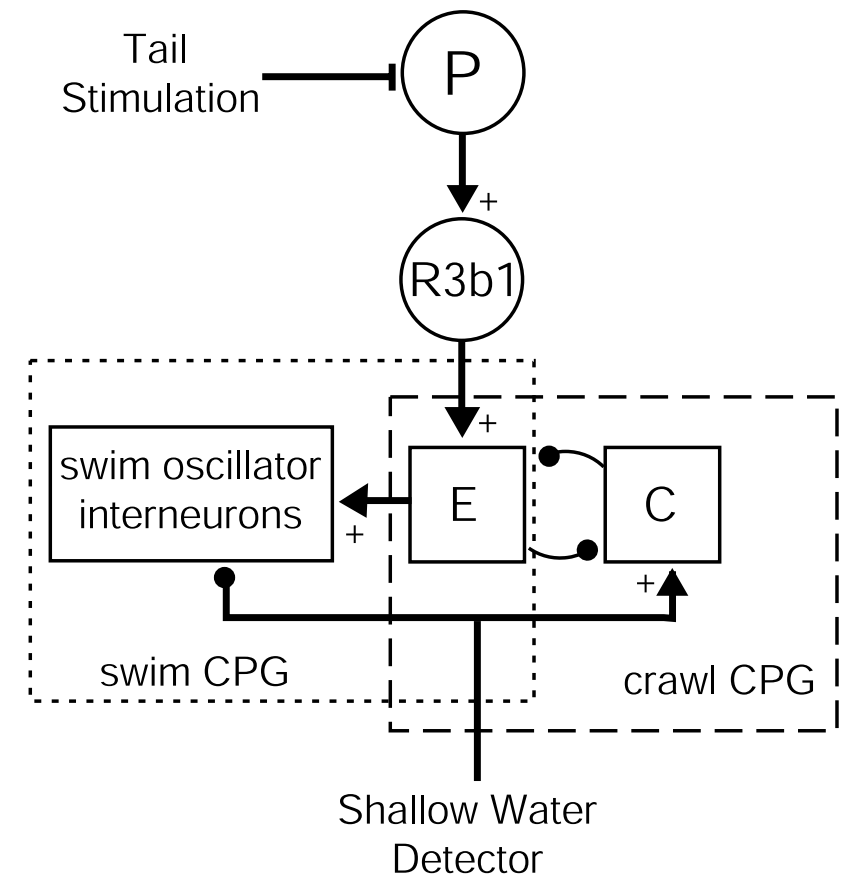

Figure 7. Working hypothesis of the behavioral effects of neuron R3b1. $P$, Pressure-sensitive cells. $E$, An elongation network that is a component of both the swim and the crawl CPGs. Filled circles indicate inhibitory interactions; filled triangles with plus signs indicate excitatory interactions.

circular motor neuron $\mathrm{CV}$, that produces elongation (Figs. $2 b, c$, $3 b, d)$. Elongation activity can either activate swim oscillator interneurons (Brodfuehrer et al., 1995b) to produce the swimming motor pattern (Fig. 3c) or interact with a contraction network (C) to produce a crawling step (Cacciatore et al., 2000) (Fig. 3b). Although the neural architecture underlying these central pattern generators (CPGs) is functionally distinct, they may share neurons whose connections are reconfigured to produce two different motor patterns (Dickinson, 1995). In intact or semi-intact animals, the selection of locomotory form depends on information about the depth of fluid around the leech's body. This information is carried in part by head brain nerves (Fig. $4 b$ ). The nature of this sensory input is unknown, but possible sources include mechanosensory stimulation by the surface of the fluid and/or substrate when the leech is in shallow water, a sensation of buoyancy when the leech is in deep water, or contact with a suitable attachment point by the front sucker. In Figure 7, the sensory input is shown as "shallow water detectors" that bias the output in favor of crawling by activating the crawl CPG and inactivating the swim CPG. The existence of such sensors is supported by evidence that when sensory inputs to the head brain are eliminated, behavior is biased toward swimming (Fig. 4b). There also might be "deep water detectors" that activate the swim CPG and inactivate the crawl CPG. In either case, intermediate fluid levels would reduce the inhibition by these sensors, resulting in net excitation of both CPGs, thereby producing the hybrid swim/ crawl motor pattern (Fig. $3 d$ ).

Results from the isolated nerve cord, in which all of the water sensors are removed, indicate that this model cannot be complete. Figure 2 shows that intracellular activation of an R3b1 neuron in an isolated nerve cord can produce either swimming or crawling, whereas the connections hypothesized in Figure 7 predict that activating R3b1 with no sensory feedback should always select the swim CPG. Which motor pattern is produced by activating R3b1 in an isolated nerve cord probably depends on the state of "spontaneous activity" in the cord when R3b1 is stimulated (Brodfuehrer et al., 1995b). We predict that there are additional sensory and modulatory pathways onto, and possibly between, the CPGs and that temporal variability in the state of these connections influences which behavior-swimming, crawling, or swim/ crawl-is produced.

\section{Behavioral modules}

Behavioral units or "modules" that can be combined in different ways to produce different behaviors have been proposed in models of spinal organization in vertebrates (Grillner, 1981; Bizzi et al., 2000). Our results indicate that such modules may be important in the construction of movement in invertebrates as well. Regardless of which locomotory behavior R3b1 elicits, the first movement that occurs after depolarization of the cell is elongation. This suggests that elongation is a component of both swimming and crawling and that R3b1 directly activates this behavioral module. Sensory input about the fluid level might contribute to activation of other modules, such that either swimming or crawling is ultimately elicited.

Evidence of modular organization also exists for other invertebrates. For example, a cerebral interneuron in Aplysia that is activated during several behaviors always elicits arterial shortening in the neck (Xin et al., 1996). Arterial shortening is a component of several neck-shortening behaviors, including head lift- 
ing, turning, and locomotion. Therefore, it appears that modular organization is a common feature of behavioral control in vertebrates and invertebrates alike.

\section{Hybrid behaviors and multifunctional neurons}

The swim/crawl behavior in the leech is similar to hybrid behaviors between scratching and stepping in turtles (Earhart and Stein, 2000) and between walking and paw shaking in cats (Carter and Smith, 1986a,b), in that one behavior is expressed only during a particular phase of the other. In the leech, swimming is expressed only during the elongation phase of crawling. This implies that there must be some overlap between the circuits controlling these behaviors such that crawling can gate the expression of swimming. A similar sort of gating is produced in the crustacean stomatogastric ganglion by presynaptic inhibition of a modulatory neuron by a gastric mill neuron. This inhibition results in the pyloric rhythm being more active during one phase of the gastric mill rhythm than during the other phase (Bartos and Nusbaum, 1997). In the leech, because elongation is a component of both behaviors, it is a likely point of intersection between the two circuits (Fig. 7). Another point of interaction may be cell 204, a segmentally iterated neuron that readily elicits swimming on activation (Weeks and Kristan, 1978) but which also oscillates during crawling (Kristan et al., 1988).

In many neuroethological studies, a single behavior is studied in isolation. Consequently, neurons involved in producing a behavior often are perceived as being dedicated to that one behavior. Our results, however, add to expanding evidence that many neurons are multifunctional, constituting portions of neural circuits shared by several behaviors (Kristan and Shaw, 1997). In Tritonia, swimming and crawling are produced by the same CPG neurons (Popescu and Frost, 2002). Note, however, that crawling in Tritonia is mediated by cilia, which generally beat while the animal is swimming, so the animal does not have to switch between the two behaviors. Experiments in Aplysia have shown that two circuits can share most elements, with the activity of only a few neurons determining which of the two behaviors is expressed (Jing and Weiss, 2001). Similarly, in the crustacean stomatogastric nervous system (STNS), different levels of activity in a single mechanosensory neuron can elicit different motor patterns from the same pattern-generating network (Combes et al., 1999). Previous work on the STNS has revealed that neural circuits are extremely dynamic: individual neurons can switch from one circuit to another, two circuits can fuse to form a conjoint rhythm, and multiple circuits can combine to form a de novo motor pattern (Dickinson, 1995; Marder, 2000). Our work in the leech has indicated that even "command-like" interneurons are not dedicated to a single behavior but that behavioral decisions are made by combinations of such interneurons. Specifically, several neurons whose activation individually causes swimming are also active during shortening (Shaw and Kristan, 1997), and as shown here, a single neuron can trigger two different behaviors. Therefore, even qualitatively different, incompatible behaviors may share decision-making interneurons.

\section{Sequential decision making}

Swimming and crawling are two forms of locomotion, the goal of which is to move an animal from place to place (Stein et al., 1986). Our results suggest that the choice to locomote is made by R3b1, independently of the decision of what form of locomotion to perform (i.e., swimming or crawling). Because electrical stimulation of R3b1 elicits crawling or swimming and because stimuli that halt locomotion hyperpolarize R3b1, it appears that R3b1 is a command-like neuron for locomotion. Because swimming, crawling, or the hybrid can be produced by stimulation of R3b1 under different environmental conditions, however, sensory information must influence which motor pattern is activated by $\mathrm{R} 3 \mathrm{~b} 1$, so a behavior appropriate for the present conditions is produced. Therefore, the decision to crawl or swim must be distributed over multiple neurons.

On the basis of our results, we propose a neural structure in which a behavioral task is selected before the form of task is selected. We envision a sequential process, in which the choice is narrowed at each step until finally a specific form of behavior is selected and produced.

Other animals also appear to make decisions sequentially. Most notably, it has been demonstrated that cockroaches, like leeches, choose to locomote independently of choosing what form of locomotion to produce. Specifically, a single neuron can elicit either flying or walking depending on whether the legs are in contact with the ground (Ritzmann et al., 1980). Our preparation has the advantage of being able to switch repeatedly among behaviors simply by changing the water levels (rather than removing the sensory structures, as in the previous study). Our results, therefore, considerably strengthen the hypothesis that using sensory information to guide output of locomotory decision centers is a common feature of behavioral organization.

Few other studies have considered decision making a multistep process. Instead, studies have focused on a single level of choice. In one model system for determining the neural basis of behavioral choice, monkeys are trained to make a saccade in different directions in response to a visual stimulus (Shadlen and Newsome, 1996; Platt and Glimcher, 1999; Gold and Shadlen, 2000; Schall, 2000, 2001; Glimcher, 2001). In these experiments, the monkey's goal is to receive a reward. The task is defined by the experimenter, for example, to make a saccade indicating the predominant direction of movement of dots. The decision being studied, therefore, is what form of the task to perform - a saccade to the left or to the right-based on the monkey's perception of the visual stimulus. Presumably, the monkey first must decide that it will perform the expected task, but nothing yet is known about how and where that decision is made.

From previous and current results, it appears that leeches decide how to respond to stimuli sequentially. At the first stage, it decides to do something (Shaw and Kristan, 1997). Next, it decides to locomote (present study). At the final premotor stage, the leech decides whether to swim or to crawl (Weeks and Kristan, 1978). Whether such a sequential mechanism is present in mammalian brains remains to be seen, but it seems unlikely that more complex decisions are made with simpler neural interactions.

\section{REFERENCES}

Bartos M, Nusbaum MP (1997) Intercircuit control of motor pattern modulation by presynaptic inhibition. J Neurosci 17:2247-2256.

Bizzi E, Tresch MC, Saltiel P, d'Avella A (2000) New perspectives on spinal motor systems. Nat Rev Neurosci 1:101-108.

Brodfuehrer PD, Burns A (1995) Neuronal factors influencing the decision to swim in the medicinal leech. Neurobiol Learn Mem 63:192-199.

Brodfuehrer PD, Friesen WO (1986) Initiation of swimming activity by trigger neurons in the leech subesophageal ganglion. I. Output connections of Tr1 and Tr2. J Comp Physiol [A] 159:489-502.

Brodfuehrer PD, Debski EA, O'Gara BA, Friesen WO (1995a) Neuronal control of leech swimming. J Neurobiol 27:403-418.

Brodfuehrer PD, Parker HJ, Burns A, Berg M (1995b) Regulation of the segmental swim-generating system by a pair of identified interneurons in the leech head ganglion. J Neurophysiol 73:983-992.

Cacciatore TW, Rozenshteyn R, Kristan Jr WB (2000) Kinematics and 
modeling of leech crawling: evidence for an oscillatory behavior produced by propagating waves of excitation. J Neurosci 20:1643-1655.

Carter MC, Smith JL (1986a) Simultaneous control of two rhythmical behaviors. I. Locomotion with paw-shake response in normal cat. J Neurophysiol 56:171-183.

Carter MC, Smith JL (1986b) Simultaneous control of two rhythmical behaviors. II. Hindlimb walking with paw-shake response in spinal cat. J Neurophysiol 56:184-195.

Combes D, Meyrand P, Simmers J (1999) Dynamic restructuring of a rhythmic motor program by a single mechanoreceptor neuron in lobster. J Neurosci 19:3620-3628.

Dickinson PS (1995) Interactions among neural networks for behavior. Curr Opin Neurobiol 5:792-798.

Earhart GM, Stein PS (2000) Scratch-swim hybrids in the spinal turtle: blending of rostral scratch and forward swim. J Neurophysiol 83:156-165.

Eisenhart FJ, Cacciatore TW, Kristan Jr WB (2000) A central pattern generator underlies crawling in the medicinal leech. J Comp Physiol [A] 186:631-643.

Esch T, Kristan Jr WB (2002) Decision-making in the leech nervous system. Integr Comp Biol, in press.

Glimcher PW (2001) Making choices: the neurophysiology of visualsaccadic decision making. Trends Neurosci 24:654-659.

Gold JI, Shadlen MN (2000) Representation of a perceptual decision in developing oculomotor commands. Nature 404:390-394.

Grillner S (1981) Control of locomotion in bipeds, tetrapods, and fish. In: Handbook of physiology (Brookhart JB, Mountcastle VB, eds), pp 1179-1236. Bethesda, MD: American Physiological Society,

Jing J, Weiss KR (2001) Neural mechanisms of motor program switching in Aplysia. J Neurosci 21:7349-7362.

Kristan WB, Stent GS, Ort CA (1974) Neuronal control of swimming in the medicinal leech. I. Dynamics of the swimming rhythm. J Comp Physiol 94:97-119.

Kristan Jr WB, Shaw BK (1997) Population coding and behavioral choice. Curr Opin Neurobiol 7:826-831.

Kristan Jr WB, Wittenberg G, Nusbaum MP, Stern-Tomlinson W (1988) Multifunctional interneurons in behavioral circuits of the medicinal leech. Experientia 44:383-389.

Lockery SR, Kristan Jr WB (1990) Distributed processing of sensory information in the leech. I. Input-output relations of the local bending reflex. J Neurosci 10:1811-1815.

Marder E (2000) Motor pattern generation. Curr Opin Neurobiol 10:691-698.
Nicholls JG, Purves D (1970) Monosynaptic chemical and electrical connexions between sensory and motor cells in the central nervous system of the leech. J Physiol (Lond) 209:647-667.

Platt ML, Glimcher PW (1999) Neural correlates of decision variables in parietal cortex. Nature 400:233-238.

Popescu IR, Frost WN (2002) Highly dissimilar behaviors mediated by a multifunctional network in the marine mollusk Tritonia diomedea. J Neurosci 22:1985-1993.

Ritzmann RE, Tobias ML, Fourtner CR (1980) Flight activity initiated via giant interneurons of the cockroach: evidence for bifunctional trigger interneurons. Science 210:209-211.

Schall JD (2000) From sensory evidence to a motor command. Curr Biol 10:R404-R406.

Schall JD (2001) Neural basis of deciding, choosing and acting. Nat Rev Neurosci 2:33-42.

Shadlen MN, Newsome WT (1996) Motion perception: seeing and deciding. Proc Natl Acad Sci USA 93:628-633.

Shaw BK, Kristan Jr WB (1995) The whole-body shortening reflex of the medicinal leech: motor pattern, sensory basis, and interneuronal pathways. J Comp Physiol [A] 177:667-681.

Shaw BK, Kristan Jr WB (1997) The neuronal basis of the behavioral choice between swimming and shortening in the leech: control is not selectively exercised at higher circuit levels. J Neurosci 17:786-795.

Shaw BK, Kristan Jr WB (1999) Relative roles of the S cell network and parallel interneuronal pathways in the whole-body shortening reflex of the medicinal leech. J Neurophysiol 82:1114-1123.

Stein PSG, Mortin LI, Robertson GA (1986) The forms of a task and their blends. In: Neurobiology of vertebrate locomotion (Grillner S, Stein PSG, Stuart DG, Forssberg H, Herman RM, eds), pp 201-216. Hampshire, UK: MacMillan.

Stuart AE (1970) Physiological and morphological properties of motoneurones in the central nervous system of the leech. J Physiol (Lond) 209:627-646.

Tinbergen N (1951) The study of instinct. Oxford: Clarenden.

Weeks JC, Kristan Jr WB (1978) Initiation, maintenance, and modulation of swimming in the medicinal leech by the activity of a single neurone. J Exp Biol 77:71-88.

Xin Y, Weiss KR, Kupfermann I (1996) A pair of identified interneurons in Aplysia that are involved in multiple behaviors are necessary and sufficient for the arterial-shortening component of a local withdrawal reflex. J Neurosci 16:4518-4528. 